\title{
Towards a Typology of Meaningful Signals and Cues in Social Robotics
}

\author{
Frank Hegel ${ }^{1,2}$, Sebastian Gieselmann ${ }^{1,3}$, Annika Peters ${ }^{1,2}$, Patrick Holthaus ${ }^{1,4}$, Britta Wrede $^{1-4}$
}

\begin{abstract}
In this paper, we present a first step towards a typology of relevant signals and cues in human-robot interaction (HRI). In human as well as in animal communication systems, signals and cues play an important role for senders and receivers of such signs. In our typology, we systematically distinguish between a robot's signals and cues which are either designed to be human-like or artificial to create meaningful information. Subsequently, developers and designers should be aware of which signs affect a user's judgements on social robots. For this reason, we first review several signals and cues that have already been successfully used in HRI with regard to our typology. Second, we discuss crucial human-like and artificial cues which have so far not been considered in the design of social robots although they are highly likely to affect a user's judgement of social robots.
\end{abstract}

\section{INTRODUCTION}

The original meaning of the term 'automaton' implies autonomous beings having the ability to move on their own [1]. For instance, Vausanson's flute and tabor player and Wolfgang von Kemepelen's famous chess player, named 'The Turk', designed in the mid 1700s, are early encounters between lifelike forms and mechanical machines. These machines invoked people's expectations due to a lifelike behaviour displayed by their appearance. Even today, social roboticists connect human-like forms and function in an attempt to develop lifelike and understandable social robots (see Figure 1).

An important objective of research in social robotics is the development of robots which are generally compelling and understandable. Therefore, roboticists generally use familiar signs to create meaningful actions. Some of these signs are understandable, because they are human-like, and other signs are interpreted correctly due to convention. To illustrate, it is likely that many people assume a blinking LED in a laptop is representing a harddisk actively working.

Faculty of Technology, Applied Informatics, Bielefeld University, Universitaetsstr. 25, 33615 Bielefeld, Germany. E-mail: \{fhegel, sgieselm, apeters, pholthau, bwrede\}@techfak.uni-bielefeld.de

Center of Excellence in 'Cognitive Interaction Technology' (CITEC) at the Bielefeld University, Germany

Research Institute for Cognition and Robotics (CoR-Lab) Bielefeld

University, Bielefeld, Germany

CRC 673, 'Alignment in Communication' at the Bielefeld

University, Germany
In addition, many of the signs implemented subconsciously into a robot have not been considered to convey any relevant information - but this is often not true for an observer. However, up to now there has not been any systematical research to which extent cues like jerky movements, sounds of actuators, or visibility of technical conjunctions have an effect on the user's judgement of robots.
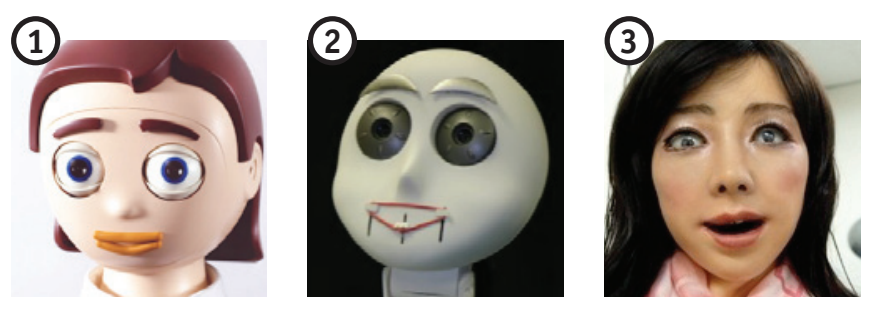

FIGURE 1. Examples of social robots with life-like forms:

(1) Flobi, (2) EMILY, (3) EVER-1

Many of the signs displayed by a robot are explicitly implemented and others are likely to be inevitable in the development of robots unless engineers, developers, and designers (in the following roboticists) invest high costs to remove or modify them. For example, if there is a need for very quick movements the actuator has a specific size, because tiny actuators are not able to move heavy parts quickly. Therefore, designing social robots often is a trade off between technical and aesthetical aspects and a management of which signals and cues are important for a specific interaction. To illustrate, it is difficult to create a tiny robot with the capability of lifting heavy weights. To make roboticists aware that from a users point of view a social robot is a semiotic entity, we discuss a first systematic analyzation towards a typology of potential signs displayed by a social robot.

After outlining relevant research in Section II, we introduce a typology of a robot's signals and cues in Section III. In Section IV, we discuss several aspects of our proposed typology. Finally, we present a conclusion and recommendations for prospective research in Section V.

\section{RELATED WORK}

Basically, a sign is defined as an entity which signifies another entity [2]. According to [3] all signs in biological communication systems are divided into signals or cues. We argue, it is generally fruitful to distinguish between signals and 
cues in social robotics as well, because such a differentiation makes it possible to understand the intended and unintended signs with regard to the technical as well as to the aesthetical design of a social robot. Most often, roboticists consider to implement specific signs (e.g., understanding and production of verbal language) to alter the user's behavior. In addition to these intended signs, a design of robots often contains unconsidered signs which may affect the user's judgement of the robot and the anticipation of the robot's behavior as well. Such distinctions lead us towards a systematical typology of human-related and artificial-related signs which are highly likely to affect expectations in HRI.

\section{A. Working Definition of Signals and Cues}

According to [3] signals are per definition signs which are acted to alter the behavior of another organism. Signals evolved, because of that effect and they are effective due to the receiver's response to certain signals. In robotics, that is, for example, a robot displaying a certain set of behaviors like verbal language or gestures to guide a user. Such signals are explicitly implemented by a roboticist to choreograph a certain scenario in HRI.

On the contrary, cues represent any feature of the world, animate or inanimate, that can be used by a receiver (i.e., in our case a user) as a guide to get certain information about the signaler (i.e., robot). In robotics many cues have not been taken into consideration to explicitly guide an interaction. That is, even if a sign is not intended to have communicative value, a robot contains naturally plenty of cues which are likely to inform a user about the robot's capabilities. To illustrate, a motor sound is a cue of an active motor; a harddisk sound is a cue representing a specific kind of action oppositional, if there is no sound or motion then users expect probably something is going wrong.

Subsequently derived from the original definitions, $h u$ man signals expressed by a robot are human-like signs to alter the behavior of another human interaction partner (e.g., verbal and nonverbal behavior). Human cues are signs which are not explicitly expressed, but contain information especially for the receiver (e.g., specific visual features about gender, age, or ethnicity). Artificial signals are explicitly implemented conventional signs (symbols) to guide a user (e.g., blinking control LEDs representing a specific action). Finally, artificial cues are any signs which convey information to an observer except of signals. Many artificial cues like, for instance, jerky movements of technical parts are not considered to carry any information also for users. Some artificial cues are explicitly considered by a roboticist to convey a specific communicative value.

\section{B. Human Signals and Cues Displayed by Social Robots}

In general, social robots should be able to communicate with humans, they should understand and even relate to humans in a personal way, and finally, they should be able to understand humans and itself in social terms [4]. Thus, one main goal is to create an automaton that appears and behaves to a certain degree intuitive during HRI [5]. However, to reach this goal, social robots need to be capable of conveying human-like signals and cues in a familiar way. Human-like signals are ideally represented by human-like displays. Specifically, the human face is probably the most variable and expressive part of the human body and as such, its signals and cues play a key role in natural interactions. The human face is composed of structural, dynamic and artificial features, all of which convey rich information about individuals. Specifically, the human face conveys information about age, sex, ethnicity, identity, fitness, and emotions [6]. Roboticists can use this repertoire of information to create social robots whose faces appear similar to human faces [7].

Human-like appearance is a human cue explicitly applied to social robots to increase the perceived human-likeness [8] in order to convey further signals like human facial expressions. Specifically, displays of emotions are often implemented to generally improve the perceived quality of interaction [e.g., 9]. Additionally, in many robots speech production and recognition has been implemented to guide the HRI - a typically human signal to design a familiar communication with a robot.

In addition, with regard to human cues, some roboticists design the robot to have a very specific human-like appearance. That is, the robot looks like a specific person having an individual appearance (i.e., gender, ethnicity, attractiveness etc.). Some of these cues also affect the perceived human traits ascribed to such robots. To illustrate, it has been shown that even within cartoon-like robots subtle hair cues significantly affect the perception of gender in terms of stereotypically female or male traits and gender related taskcapabilities [10].

The same ist true for the individually perceived attractiveness. A vast body of research documents that people attribute more positive traits to attractive people than to unattractive ones. In social psychology, this bias is known as the 'attractiveness halo' [11]. To illustrate, attractive humans are commonly judged as warmer, kinder, stronger, more sensitive, interesting, poised, modest, sociable, and outgoing. It has been shown that even babies prefer playing with attractive than with unattractive puppets [12]. Importantly, several authors [e.g., 13, 14, 15] suggested that an attractiveness bias is generally also applicable to objects and interfaces.

Additionally, anthropomorphism is of importance due to the fact that the more specific human-like signals and cues were used to aesthetically design the robot the more the robot is likely to be anthropomorphized by a human observer [16]. Anthropomorphism entails attributing human-like properties, characteristics or mental states to real or imagined nonhuman agents and objects.

The key role of human-likeness with regard to anthropomorphism has been demonstrated in a study by Krach et al. [17]. These authors conducted a fMRI study with three different robot targets which differed in their degree of humanlike appearance. Participants' brain activity was measured during the interaction with these robots. The results showed 


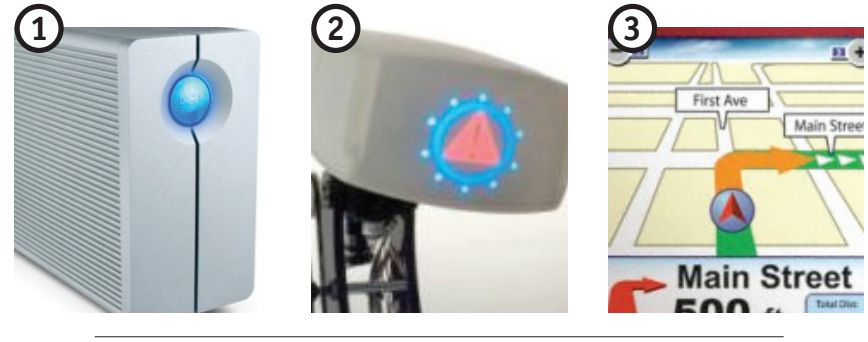

FIGURE 2. Examples of artificial signals: (1) power LED,

(2) facial display of the robot AIDA, (3) GPS interface in a mobile phone

that the degree of human-likeness had a significant effect on participants' cortical activity associated with Theory of Mind (ToM) and their judgments of the robots. Summing up, the more human-like an interaction partner appeared, the more do participants speculate implicitly about the robot's intentions.

\section{Artificial Signals and Cues in Social Robotics}

Principally, all conventional symbols and artificial interfaces used in products which automatically represent dynamically a state are per definition artificial signals that alter the action of an user. For example, artificial signals like blinking or colored LEDs are often used to report the machine's actual state. Furthermore, [18] found that users prefer to have visual indicators on the mobile robot BIRON, to signal them in which direction it is going to drive next. Additionally, in some cases roboticists implemented dynamic interfaces dis- playing a conventional symbol are artificial signals (see Figure 2, image 2).

Often, designers explicitly consider artificial cues to improve a product's perceived quality, but particularly in social robotics, artificial cues are rarely taken into account to improve the robot's perceived quality. In the field of car design, for instance, sound designers at Porsche explicitly try to improve the perceived quality of the car's functions by modulating sounds of motors, clicks, sound intensity, and so on [19]. Also visual signs are commonly used to mark specific functions. For instance, mechanical joints of robots are often visually emphasized to especially display the mechanical function (i.e., form follows function).

Only little research on a social robot's cues has been explicitly taken into account. For instance, there is evidence that unattractive cues in human-like social robots elicit some kind of unpleasantness. This phenomenon is widely known as the 'uncanny valley' hypothesis [20] which is particularly true for realistic robot faces with specific abnormal facial features (e.g., with regard to eye size). This suggests that the human visual system is highly sensitive to cues indicating human-likeness [21]. Most often, designing a robot is a trade off between mechanical architecture and the robot's appearance. Due to the fact that many of the robots are research prototypes instead of finished products, roboticists primary concentrate on the robot's technical functions rather than on aesthetic functions including an attractive appearance and an absence of abnormal perceived facial features.

TABLE 1. Examples of signals and cues in human communication (top) and artificial signals and cues (bottom)

\begin{tabular}{|c|c|c|c|}
\hline \multicolumn{4}{|r|}{ human signals \& cues } \\
\hline & perception & example & possible indications \\
\hline \multirow[t]{3}{*}{ signals } & \multirow[t]{2}{*}{ appearance } & emotional displays & nonverbal emotional communication \\
\hline & & clothing and decoration & communication of social roles \\
\hline & auditive & verbal language & human communication / natural language skills \\
\hline \multirow[t]{6}{*}{ cues } & \multirow[t]{2}{*}{ appearance } & facial qualities & specific human traits (e.g., domincance, submissiveness, social warmth etc) \\
\hline & & body size & dominance (e.g., roboticists often use small body sizes to mediate submissiveness) \\
\hline & auditive & unintentional prosody & uncertainty \\
\hline & tactile & temperature & illness \\
\hline & olfactory & smell & illness or personal hygiene \\
\hline & motion & breath & subject is alive \\
\hline \multicolumn{4}{|c|}{ artificial signals \& cues } \\
\hline & perception & example & possible indications \\
\hline \multirow[t]{3}{*}{ signals } & appearance & LED & power and activity \\
\hline & motion & robot's gaze & joint attention \\
\hline & auditive & beep sounds & convention (e.g., confirmation or error) \\
\hline \multirow[t]{5}{*}{ cues } & appearance & emphasized conjunctions & communication of stability and robustness \\
\hline & auditive & harddisk working sound & indicating the machine works \\
\hline & tactile & high temperature & overheating \\
\hline & olfactory & smell & disfunction, overheating \\
\hline & motion & jerky movements & indicating low quality \\
\hline
\end{tabular}




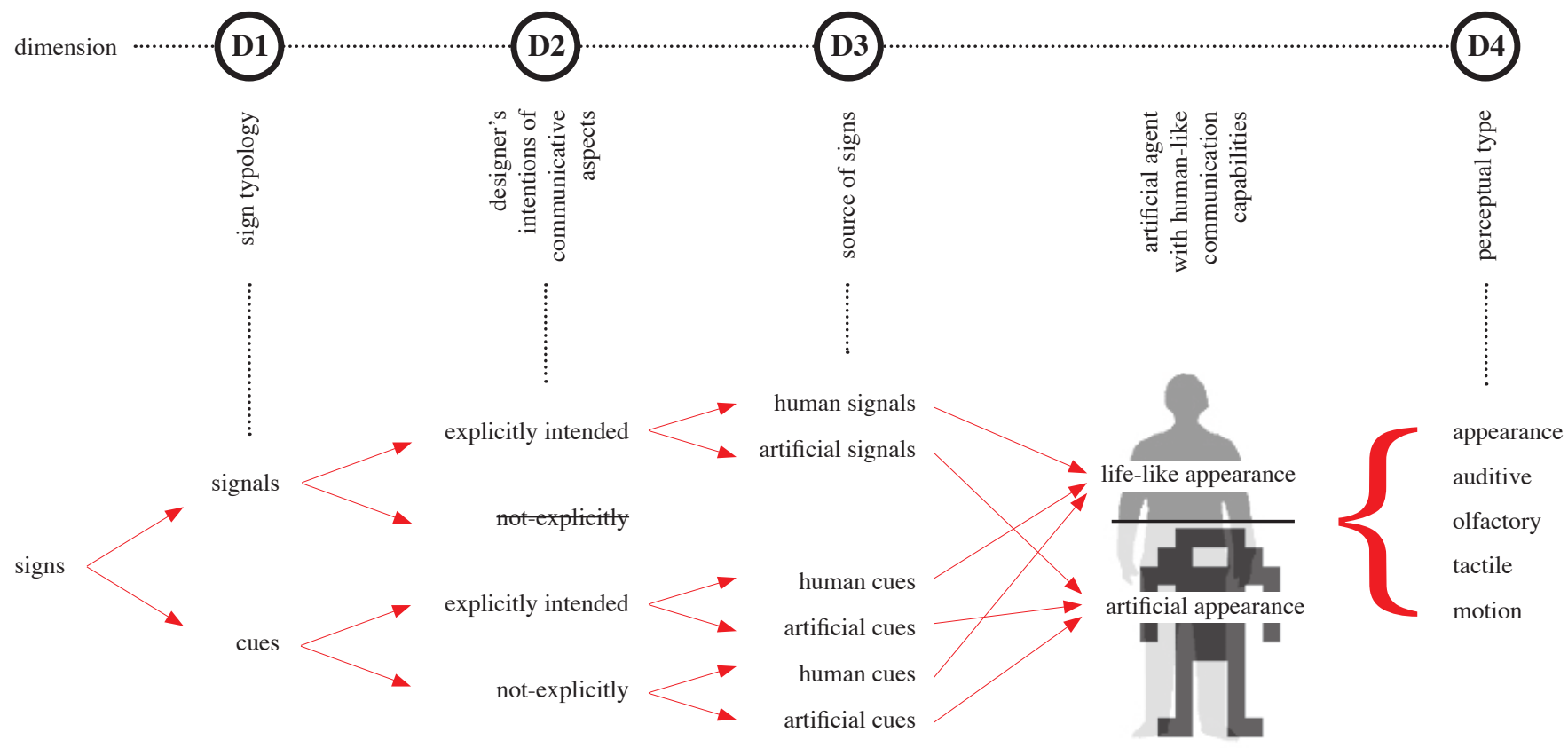

FIGURE 3. Towards a typology of human and artificial signals and cues for roboticists and their robots. All signs expressed by a social robot are divided into four dimensions (signals vs. cues, explicitly vs. implicitly implemented, human vs. artificial signs, and the use of different perceptual channels)

\section{TyPology OF Signals AND CUES In RoBOtics}

In the field of social robotics, roboticists usually apply human signals and cues to facilitate the social interaction between human and machine, because many of the human-like signals and cues used in robotics are familiar to users (see examples in Table 1). Artificial signals are usually used to report the product's actual state to users. Although artificial cues might communicate specific qualities of a product, they are rarely considered in the development of social robots probably due to the fact that most of today's social robots are research prototypes.

As argued above, with regard to a typology of signs in HRI we first of all distinguish between signals and cues (see Figure 3). On a second dimension, we differentiate whether the signs used for the robot are taken into consideration by the roboticist. Actually, all signals used to alter the behavior of a user are intended to do so. Per definition, there are no signals which are not explicitly intended by a roboticist. The same is not true for cues due to the fact that some cues are explicitly considered to have a communicative value and others are not. On a third dimension, we differentiate whether the signals and cues are human-like or artificial. For example, a 'not explicitly considered human cue' is a technical robot design that randomly appears similar to a human face. Finally, all the signs are perceived differently. That is, signals and cues in robots are sensed visually (appearance and motion), auditive, olfactory, or tactile by a human user.

To summarize, as illustrated in Figure 3, we classify all potential signs used in a robot into four dimensions: (D1) signals vs. cues, (D2) explicitly vs. implicitly used (D3) human vs. artificial signs, and (D4) different perceptual channels.

\section{Discussion: Costs, DeCEPTION, AND RELIABILITY}

Figure 3 displays the typology of signals and cues with different perspectives or dimensions. The typology is divided into four dimensions. In contrast to a typology of signals and cues in human-human interaction (HHI), the typology in HRI has another dimension. Here, dimension two represents the designer's point of view, which makes the typology more complex compared to HHI. A designer has the possibility to explicitly modify the robot's signals and even cues by design. A robot's appearance can include particularly intended cues, which should be received as if they were not controlled at all. Additionally, there are cues which are not explicitly intended by the designer. Hence, this adds not only to the typology's complexity but also to the costs, which have to be spent to make the signals and cues perceivable and reliable. Furthermore, with regard to costs the special case of deceptive signals is discussed as such a social robot is particularly a deception of a lifelike interaction partner. Inseparably, the reliability of a robot's signals as well as cues and thus the consequences for human-robot interaction in general are taken into account.

\subsection{Costs of Honest Signals}

Generally, building a social robot as such is a costly undertaking. In particular, ensuring that signals and cues are received the way they should, is even more costly as it requires a lot of time and manpower to pursue the circle of design: planning, testing, and refining. In nature, cues do not have any costs as they are innate and unintended. Therefore, they can hardly be deceived [3], but cues of a robot can be as costly as signals - or in certain cases even more costly. 
TABLE 2. Costs of Designing a Robot's Signals

\begin{tabular}{|l|l|l|}
\hline costs for robots & efficacy costs & strategic costs \\
\hline $\begin{array}{l}\text { preperation } \\
\text { (material) }\end{array}$ & $\begin{array}{l}\text { minimal costs of } \\
\text { material }\end{array}$ & $\begin{array}{l}\text { quality of material } \\
\text { (e.g., joints, skin, } \\
\text { sensors) }\end{array}$ \\
\hline $\begin{array}{l}\text { preperation } \\
\text { (manpower) }\end{array}$ & $\begin{array}{l}\text { necessary hardware } \\
\text { design (appearance) }\end{array}$ & $\begin{array}{l}\text { hardware design (ap- } \\
\text { pearance) }\end{array}$ \\
\cline { 2 - 3 } & $\begin{array}{l}\text { basic software design } \\
\text { (behavior, perception) }\end{array}$ & $\begin{array}{l}\text { software design } \\
\text { (behavior, perception, } \\
\text { exception handling) }\end{array}$ \\
\hline $\begin{array}{l}\text { production } \\
\text { (ad-hoc costs) }\end{array}$ & $\begin{array}{l}\text { costs to display the } \\
\text { signal }\end{array}$ & $\begin{array}{l}\text { additional costs to } \\
\text { design the signal more } \\
\text { reliable }\end{array}$ \\
\hline
\end{tabular}

In Table 2 costs for designing a social robot (preparation) and production of signals and cues are shown. According to [22], the costs are divided into efficacy and strategic costs. Efficacy costs represent the minimal costs with regard to functional robots. Strategic costs are invested to improve the robot's quality in terms of displaying reliable signals and cues.

Efficacy and the strategic costs interplay with each other and are not always comparable with costs in HRI. For example, the natural way for a human to walk might be the way with the lowest costs, but for a robot it is less efficient and more costly to walk like a human.

Furthermore, we divided costs for a robot into preparation costs and production costs. That is, money has to be spent before a human-robot interaction is feasible. These costs are additionally divided into material costs as such and the work which roboticists have to accomplish to make the hardware do a certain action. Moreover, there are energyrelated costs (e.g., electricity) for producing the signal within interactions.

\subsection{Deception of Signals and Cues}

Another aspect with regard to costs is the deception of signals and cues. Deceptive signs are any such signals and cues which are dishonest. With deceptive signals a sender tries to actively spread false information about oneself, while deceptive cues are typically misleading features of an agent. In any case, the receiver is informed about a quality that the sender does not have [3].

Besides the costs for sending honest signals, the use of deceptive signs potentially involves additional costs due to punishments. These costs affect signaler, receiver and also other signalers. For instance, if a lie has been detected by a receiver, the credibility of a signaler might be negatively affected. As a consequence, this results in a decrease of sender's reliability. In addition, similar information conveyed by other senders might also be less reliable, which introduces costs for these senders no matter if the message is honest or not. The receiver's costs do not only originate from a believe in the false signal but also from any action that has to be taken to not believe in a false signal again. Therefore, deceptive signals as such are unreliable and only occur if the sender's
TABLE 3. Relation between expectation and reliability

\begin{tabular}{|l|l|l|}
\hline & expectation & reliability \\
\hline deception detected & mismatch & decrease \\
\hline deception accepted & match & increase \\
\hline
\end{tabular}

costs do not outweigh the benefits. For example, humans use make-up and plastic surgery to appear younger, healthier, or more attractive. The same is true in the world of animals, specific butterflies mimic other animals (e.g., hornets) or are colored as if they were poisonous to avoid being eaten by potential predators. Specifically, in the case of the butterfly, these false signals obviously target at the benefit of a longer life and thus more chances for reproduction. The costs of being caught are enormous and most often result in becoming the next meal for a predator. In the case of a human using make-up, the final goals may be similar. However, in contrast to the butterfly, the costs of the delusion being detected are not as high as for the butterfly.

Highly anthropomorphic robots are designed to appear and to behave human-like. All the human-like signals and cues used in anthropomorphic designed robots are deceptions. That is, any signal and cue which simulates a human being is dishonest in its nature. Explicitly human-like designed gaze, breath, or voice are typical examples for deceptive signals and cues conveyed by social robots.

This has several consequences regarding the design of human-like social robots. With every single mechanical part and behavior aimed at human-likeness comes a potential loss of credibility and, consequently, a decrease of the robot' reliability.

In general, humans are aware of the fact that a robot itself is naturally not a human being. Even though they tend to interpret human-like movements as social and communicative signs, they detect these signals often as dishonest (i.e., non-human). Therefore, care has to be taken that there are no direct consequences in terms of punishments (like in the butterfly example) due to incredibly signals or cues. Not only the unmasked signals and cues would be affected, but additionally further signals and cues displayed by the robot would probably be judged as less credible.

Ultimately, it is the roboticist's task to create a semioticrelated structure of the robot's signs that will be accepted by users. If signals and cues displayed by a social robot are accepted they probably will not violate a user's expectations of what tasks a robot is or not able to perform credibly [23]. See Table 3 for the relation between expectation and reliability of the robot.

\subsection{Implications in Social Robotics}

Why should roboticists consider signals and cues in social robotics? First, humans automatically anthropomorphize robots - even if robots do not explicitly appear human-like people most often ascribe robots to have human-like traits. For instance, if roboticists apply verbal language or gestures 
to their robots they use (consciuosly or not) signals and cues typically used by humans in their everyday life. Analogous to Watzlawick [24] in human communication, robots cannot not present any cues. For a roboticist, it is impossible to not convey any information of the robot to an interpreter.

Second, the more human-like a robot appears the more people anthropomorphize it. Namely, it has been shown that humans cognitivelly [17] as well as emotionally [25] ascribe a robot to have more human-like traits if the robot appears more human-like as well. According to the familiarity hypothesis [26], individuals draw anthropomorphic inferences, because it allows them to explain things we do not understand in terms that they do understand - and what we understand best is ourselves as human beings. Consequently, if a robot appears human-like to a certain degree the quality of human signals and cues should be taken into account to design predictable interactions.

Third, the aesthetic form of robots always promises specific actions due to perceived affordances. Hence, people have specific expectations and mental models about the robot's future actions. Specifically, [23] found that people in the case of mismatches rebuild their mental models of the robot's behaviors. This is a problem when there generally exists a mismatch between the robot's appearance and its function. Accordingly, people are likely to become confused with regard to the reliability of the robot's actions if it is not able to fulfill the people's expectations.

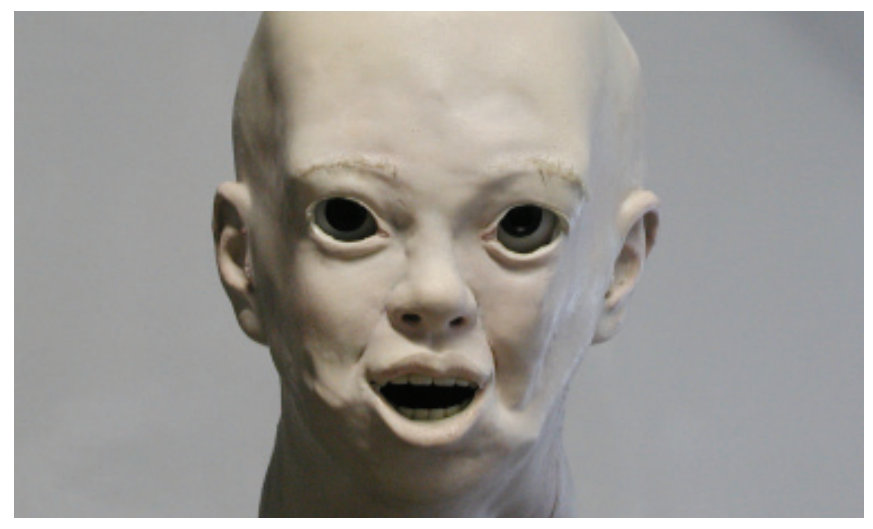

FIGURE 4. The anthropomorphic robot Barthoc Jr.

Especially in social robotics, the consideration of artificial cues have rarely been taken into account although they are highly likely to affect the judgement of robots. To illustrate, the anthropomorphic robot Barthoc Jr. (see Figure 4) was created to appear like a child-like interaction partner. Nevertheless, due to unexpected lacks in the manufacturing process participants perceived Barthoc Jr. to have abnormal facial features. Consequently, participants jugded the robot to have less capabilities than it actually has [27].

Furthermore, even when designing a robot without an effort to have a human-like appearance, artificial cues should be taken into consideration in the industrial design process. To illustrate, according to Dieter Rams [28], the wellknown industrial designer of BRAUN, something that does not indicate something else should simply not be there, because something that does not have any specific meaning for a user results in incomprehensibility of the product. Indeed, good design should be usefull, understandable, unobtrusive, honest, long-lasting, thorough, environmentally friendly, and as little design as possible.

Finally, such a typology of signals and cues in robotics like introduced in Section III supports to systematically explore the effects of a social robot's appearance and behaviors. When developing social robots roboticists try to anticipate the human-robot interaction with regard to the user's mental model of the robot. Thus, the more facts are known about such signals and cues the easier it is for a roboticist to focus on what the robot is actually communicating via form and function. Additionally, it is probably easier to avoid unwanted effects of negative judgements if a roboticist has in mind the essential meanings of robot-related visual as well as behavioral signals and cues.

\section{CONCLUSION}

In this paper we presented a first conceptualization of a typology with regard to a social robot's potentially displayed signs. We mainly distinguished between signals and cues acted by humans and social robots. Moreover, human signals and cues are likely important to be conceptualized in social robotics due to the fact that roboticists most often use such signs to create a meaningful content.

Signals in robotics are explicitly implemented by roboticists to coreograph the human-robot interaction in a specific way. We distinguish between human signals and artificial signals. Human signals are familiar human-like signals implemented in robots to facilitate the understanding of a social robot's action. Artificial signals are conventional signs to inform a user about a robot's functional state.

Furthermore, explicitly considered human and artificial cues are implemented to improve a user's perceived qualities of the social robot. Explicitly implemented human cues are, for instance, human-like faces which are babyfaced or attractive. Explicitly implemented artificial cues are any modified artificial features of a robot (e.g., industrial design).

Finally, not considered human and artificial cues are any cues which are not conceptualized in the design of a robot. To illustrate, unattractive faces with abnormal human-like features are such human cues in robots. Not considered cues might have unwanted effects with regard to a user's judgement of a social robot.

The quality of signals and cues in social robots is highly likely to influence the reliability of a robot's displayed signs. Therefore, a discussion if or not implementing specific signals and cues in robots is a trade off among reliability and costs. Costs are on the one hand preperation costs (e.g., complexitiy of a mechanical construction) and on the other hand production costs (e.g., power to display the sign). Moreover, in the consideration of signals and cues deception of such is 
a crucial aspect. Particuarly, human-like signals and cues are always per definition deceptive, because a robot is an artificial entity. Subsequently, the implementation of human-like signs should be considered carefully. To illustrate, if a robot is conceptualized to appear highly anthropomorphic without the investigation of any preperational costs in the quality of anthropomorphic features the neglected human-likeness is likely to negatively affect the users' judgement of the robot (e.g., uncanny valley effect). In this respect, a large scaled typology generally ought to be used by roboticists as a tool to minimize a user's negative judgements about the social robot. An indicator has a value when it is indicating something. But if it is not indicating something, it should not be there or at least not indicating a negative value randomly.

\section{ACKNOWLEDGEMENTS}

This work has partly been funded by the German Research Foundation (DFG) within the CRC 673 'Alignment in Communication' and by the German Aerospace Center (DLR) with funds from the Federal Ministry of Economics and Technology on the basis of a decision of the German Bundestag under grant number 50 RA 1023. Furthermore, we gratefully acknowledge the support by the 'CIT-EC' and the 'CoR-Lab' at Bielefeld University.

\section{REFERENCES}

[1] H. Jestram (2000). 'Mythen, Monster und Maschinen, Der künstliche Mensch im Film'. Teiresias-Press, Cologne, Germany.

[2] C.S.Peirce (1931). The Collected Papers of Charles Sanders Peirce. (Ed. C. Hartshorne, P. Weiss \& A.W. Burks). Cambridge, MA, Harvard University Press, USA.

[3] J. Maynard Smith and D. Harper (2003). Animal Signals. Oxford University Press.USA.

[4] C. Breazeal (2002). Designing Sociable Robots. Cambridge, MA: MIT Press, USA.

[5] F. Hegel, C. Muhl, B. Wrede, M. Hielscher-Fastabend, and G. Sagerer (2009). Understanding Social Robots. In the Proceedings of the Second International Conferences on Advances in Computer-Human Interactions (ACHI), Cancun, Mexico.

[6] L.A. Zebrowitz (1997). Reading faces: Window to the soul? Boulder, CO: Westview Press, USA.

[7] M. Blow, K. Dautenhahn, K. Appleby, C.L. Nehaniv, and D. Lee (2006). Perception of robot smiles and dimensions for humanrobot interaction design. In the Proceedings of the 15th IEEE International Symposium on Robot and Human Interactive Communication (RO-MAN), Hatfield, UK, S.469-474.

[8] C.F. DiSalvo, F. Gemperle, J. Forlizzi, and S. Kiesler (2002). All robots are not created equal: the design and perception of humanoid robot heads. In DIS '02: Proceedings of the Conference on Designing interactive systems, pp. 321-326, New York, NY, USA.

[9] F. Eyssel, F. Hegel, G. Horstmann, and C. Wagner (2010). Anthropomorphic inferences from emotional nonverbal cues: A case study. In: 19th IEEE International Symposium in Robot and Human Interactive Communication (RO-MAN). Viareggio, Italy.
[10] F. Eyssel and F. Hegel (accepted). (S)he's got the look: Gender stereotyping of social robots. Journal of Applied Social Psychology.

[11] K. Dion, E. Berscheid, and E. Walster (1972). What is beautiful is good. Journal of Personality and Social Psychology, Vol. 24 (3), pp. 285-290.

[12] J.H. Langlois, L.A. Roggman, and L.A. Ricser-Danner (1990). Infants Differential Social Responses to Attractive and Unattractive Faces. Developmental Psychology, 26, pp. 153-159.

[13] D.A. Norman (2004). Emotional Design: Why We Love (Or Hate) Everyday Things. New York: Basic Books, USA.

[14] N. Tractinsky, A.S. Katz, and D. Ikar (2000). Interacting with computers 13(2): pp. 127-145.

[15] A.F. Monk and K. Lelos (2007). Changing only the aesthetic features of a domestic product can affect its apparent usability. In A. Venkatesh et al. (ed.s) Home informatics and telematics: ICT for the next billion. Proceedings of HOIT 2007, Chennai, India. New York: Springer, pp. 221-234.

[16] N. Epley, A. Waytz, and J.T. Cacioppo (2007). On Seeing Human: A Three-Factor Theory of Anthropomorphism. Psychological Review 114, pp. 864-886.

[17] S. Krach, F. Hegel, B. Wrede, G. Sagerer, F. Binkofski, and T. Kircher (2008). Can machines think? Interaction and perspective taking with robots investigated via fMRI. In PLoS ONE, 3(7), July 2008. doi:10.1371/journal.pone.0002597.

[18] A. Peters, T. Spexard, P. Weiß, and M. Hanheide (in press). Hey robot, get out of my way. In B. Gottfried and H. K. Aghajan (editors), Behavior Monitoring and Interpretation. Well-Being.

[19] See the German Magazine Spiegel online: http://www.spiegel. de/auto/aktuell/0,1518,444498,00.html

[20] M. Mori (1997). The Buddha in the Robot. Charles E. Tuttle Co.

[21] J. Seyama and R.S. Nagayama (2007). The uncanny valley: Effect of realism on the impression of artificial human faces. Presence. Teleoperators and Virtual Environments, 16(4), pp. 337351.

[22] M.S. Dawkins and T. Guilford (1991). The corruption of honest signalling. Anim. Behav 41, pp. 865-873.

[23] M. Lohse (in press). The role of expectations and situations in human-robot interaction. New Frontiers in Human-Robot Interaction: John Benjamins Publising Company.

[24] P. Watzlawick, J.H. Beavin, and D.D. Jackson (1968). Pragmatics of human communication: a study of interactional patterns, pathologies, and paradoxes", Faber, London, UK.

[25] L.D. Riek, T. Rabinowitch, B. Chakrabarti, and P. Robinson (2009). How Anthropomorphism Affects Empathy Toward Robots. In Proceedings of the 4th ACM/IEEE International Conference on Human-Robot Interaction (HRI 09), San Diego, CA, USA.

[26] S.E. Guthrie (1997). Anthropomorphism: A Definition and a Theory. In: R. W. Mitchell et al., 'Anthropomorphism, anecdotes, and animals'. Albany: State University of New York Press., USA.

[27] M. Lohse, F. Hegel, A. Swadzba, K. Rohlfing, S. Wachsmuth, and B. Wrede (2007). What can I do for you? Appearance and Application of Robots. Annual Convention of the Society for the Study of Artificial Intelligence and Simulation of Behaviour (AISB'07). Newcastle upon Tyne, GB.

[28] K. Klemp and K. Ueki-Polet (2010). Less and More: The Design Ethos of Dieter Rams. Die Gestalten Verlag, Berlin, Germany. 